\title{
Girolamo Muzio, letterato Capodistriano
}

\author{
Nives Zudič Antonič, Anita Dessardo
}

In questo articolo verrà presentata l'opera di Girolamo Muzio, autore capodistriano di famiglia e d'affetti. Muzio era un uomo che oggi potremmo definire "di regime”, allineato con le strutture di potere. Infatti, verso la metà del Cinquecento, si trovò nella tipica posizione di servitore e di letterato, quando oramai la corte diventava sempre più centro del potere politico, mettendo così in crisi la figura dell' intellettuale, visto come maestro di civiltà. Nell'articolo viene illustrata la sua ampia produzione letteraria che comprende diversi ambiti: da quello religioso, alla questione della lingua e alle regole del comportamento.

Parole chiave: Girolamo Muzio, Controriforma, letterati capodistriani, il "martello degli eretici", Il Duello.

V članku so predstavljena dela Girolama Muzia, avtorja, ki izhaja iz koprske družine in je na Koper bil zelo navezan. Muzio je bil osebnost, ki ga lahko danes imenujemo » človek režima«, naklonjen takratni oblasti. Sredi i6. stoletja se uveljavi v vlogi dvornega privrženca in učenjaka, prav v času, ko je dvor vedno bolj postajal sedež politične moči. Tako je žal zastrt lik izobraženca kot učitelja civilizirane družbe. Članek predstavlja ves avtorjev literarni opus, ki zajema različna področja: od verskih do jezikovnih vprašanj in tudi pravil olike v družbi.

Ključne besede: Girolamo Muzio, protireformacija, koprski učenjaki, »kladivo za krivoverce «,Dvoboj.

$\mathrm{N}$

el vasto panorama degli autori istriani o che si considerarono tali, non si può non parlare di Girolamo Muzio, capodistriano di famiglia e d'affetti. A molti, purtroppo, sconosciuto o poco considerato. Si tratta di un autore ingiustamente sottovalutato che paga il suo allineamento alla curia e la sua lotta alla riforma. Il "martello degli eretici", come veniva chiamato, è una figura che a prima vista potrebbe sembrare meno affascinante del Vergerio e di coloro che si fecero carico della riforma protestante. Muzio era un uomo che oggi potremmo definire "di regime", allineato con le strutture di potere, in pratica può essere definito un cortigiano.
Infatti, verso la metà del Cinquecento, si trovò nella tipica posizione di servitore e di letterato, quando oramai la corte diventava sempre più centro del potere politico, mettendo così in crisi la figura dell' intellettuale. Visto come maestro di civiltà, Muzio svolse diverse funzioni di segretario al servizio dell'uno o dell'altro signore, sempre però subordinato al potere assoluto. Girando per le corti e frequentando molta gente, divenne particolarmente esperto in materia cavalleresca, così che nobili di tutta l'Europa lo consultarono per districare svariate querele.

La sua produzione letteraria fu molto ampia. Si cimentò in diversi campi, scrivendo sulla religione, sulla questione della lingua - che in 
quel periodo rivestiva un ruolo di primaria importanza - e sulle regole del comportamento. Coltivò, inoltre, la lirica, la forma epistolare, tentando anche l'epica che poi abbandonò. L'opera che lo rese famoso in tutta l'Europa fu il trattato intitolato il Duello.

Nonostante la sua cospicua produzione, la notorietà raggiunta e il buon grado di conservazione delle sue opere, sono stati in pochi ad occuparsi della figura di Girolamo Muzio.

\section{L'inquieto Cinquecento istriano}

\section{Il Cinquecento mise in luce alcune delle figure} più significative e d'alto livello morale, nonché intellettuale, del panorama istriano. Queste rivestirono un notevole ruolo nelle vicende politiche ed ecclesiastiche di quel periodo non solo a livello regionale, ma anche in un contesto più ampio. La loro abbondanza talvolta ci fa mettere in secondo piano personaggi che meriterebbero ben altra attenzione. Si deve rilevare che la vita spirituale istriana non si può certo esaurire nello studio di figure come Pier Paolo Vergerio il Giovane, Baldo Lupatino, Flaccio Illirico o Pietro Bonomo, come sembrerebbe dagli studi finora pubblicati in materia.

Sin dall'antichità, il sentimento che dominò fra gli istriani fu il desiderio, o meglio il bisogno spirituale di libertà, per il quale seppur sottomessi, combatterono più volte. Non fu solo la Riforma, l'unico elemento che fece scaturire nuove idee, ma è necessario considerare pure il manifestarsi di tutti quei problemi culturali, politici, economici e religiosi, che emersero ben prima. L'Umanesimo fu così il mezzo ideale per un riscatto non solo culturale, ma anche civile. Fu questo, uno dei motivi per cui gli umanisti istriani furono numerosi.

Esaminando attentamente i documenti ecclesiastici e politici dell'epoca, è evidente come nel

La Biblioteca centrale di Capodistria conserva un'edizione del Duello del 1585 .

2 Fulvio Salimbeni. "Fonti e studi sulla storia religiosa dell' Istria nel XVI secolo," in L'umanesimo in Istria, cur. Vittore Branca e Sante Graciotti (Firenze: Olschki, 1983), 167.

3 Francesco Semi, Istria e Dalmazia. Uomini e tempi. Istria e Fiume (Udine: Del Bianco, 1991).
XVI secolo si evolse, impetuosamente, quell'irrequietezza che si trovava alla base della vita istriana, non solo nel Quattrocento e all'inizio del Cinquecento, ma già alla fine del Trecento. ${ }^{4}$

La storiografia istriana ha creato un'immagine della storia moderna "tanto compatta e monolitica, tutta veneziana e italiana che, per quanto fascinosa e suggestiva, è decisamente sviante e fuorviante; e l'adesione popolare alla Riforma protestante è la prova evidente d'un malcontento latente, inconfessato, in parte inconscio contro la Dominante, anche se si devono ammettere" molte altre concause che hanno spinto a tale scelta di constatazioni dei valori ufficiali.

In ogni modo, osservandolo nel suo insieme, il quadro della letteratura istriana del Cinquecento offre un panorama sociale e culturale interessante e apparentemente contraddittorio: da un lato scrittori protestanti, come Vergerio il Giovane, Flaccio, Lupatino e fra Giulio Morato da Capodistria, dall'altro invece Girolamo Muzio Giustinopolitano, difensore della Curia romana e definito "martello degli eretici", il cittanovese Antonio Pantera, capodistriani come Giovanni Zarotti e Vincenzo Metelli. ${ }^{6}$

Nonèda trascurareche Capodistriafun importante centro culturale, dove, già nel $\mathrm{I} 478$, sorse un'accademia, la Compagnia della Calza, che dopo aver abbandonato gli eserciti cavallereschi dei suoi primi anni, si trasformò ben presto in una società letteraria, che ai tempi della riforma mutò nome in Desiosi e proibì ai suoi iscritti qualsiasi discussione su argomenti religiosi. A questo punto il Muzio accusò tutti i membri d'eresia e di convivenza con il Vergerio. Non si sa con esattezza se questo fatto fosse vero o meno, dimostra però la divisione dell'ambiente istriano: da una parte innovatori, dall'altra, conservatori. Lo stato attuale degli studi in materia non è ancora riuscito a chiarire se anche nelle altre cittadine, a Pirano, a Pola, o altrove, dovunque esi-

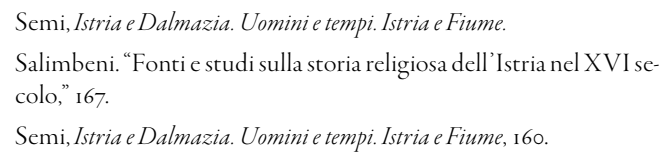


stessero accademie, succedeva lo stesso che fra $\mathrm{i}$ Desiosi. Le beghe capodistriane però sembrano indicare un certo stato d'animo caratteristico.

\section{La Controriforma in Istria}

In Istria, dominata in gran parte dalla Repubblica di Venezia e in parte dagli Arciduchi d'Asburgo, ci fu un forte influsso del luteranesimo. Le continue lotte fra l'Impero e Venezia devastarono l'Istria, influendo in modo negativo sull'andamento economico, aggravato anche dalle epidemie e dalle scorrerie dei Turchi. Le idee luterane si propagarono in questo territorio mediante sacerdoti locali e tramite predicatori luterani che giungevano da altre province. Intorno al 1520 aderivano alla Riforma non solo letterati ed ecclesiastici, ma persino donne e bambini. Questo entusiasmo cominciò in una specie d'Accademia per prepararsi, successivamente, in tutta la provincia conquistando sia le classi colte sia quelle popolari. Ben presto dalle stamperie di Venezia, prima che la "Santa Inquisizione" potesse imporre il suo veto, varcarono il confine gli scritti protestanti, che non si fermarono in Istria, ma si diffusero anche lungo la Dalmazia. Anche il vescovo di Trieste, Pietro Bonomo, ebbe una dose di responsabilità nell'importazione della "merce sassonica".

In Istria la Riforma diede quei frutti che altrove scarseggiavano, ma l'Inquisizione e le vicende politiche sfatarono le rosee prospettive, così la penisola fu costretta a chinarsi al gioco delle vecchie tradizioni. Nella seconda metà del XVI secolo l'eresia protestante si sviluppò rapidamente, e contemporaneamente si sviluppò anche la lotta dell'Inquisizione romana per contrastarne il passo. Primož Trubar svolse un ruolo importante nella diffusione dell'eresia luterana e anche Pier Paolo Vergerio il Giovane, seppur in Germania, teneva d'occhio il Friuli, tentando di convincere i giovani friulani a partire alla volta della Germania. Pier Paolo Vergerio, divenuto vescovo di Capodistria, tornò in patria nel 1544

Semi, Istria e Dalmazia. Uomini e tempi. Istria e Fiume, I6 I.

8 Antonio Miculian, "Il Santo Ufficio e la riforma protestante in Istria," in Atti del Centro di ricerche storiche, Vol. XI (Rovigno-Trieste: Centro di Ricerche Storiche di Rovigno, 1980-8I), 215-230. e nel giro di pochi anni, con l'aiuto anche del gruppo ereticale piranese, diffuse segretamente il luteranesimo in tutta l'Istria. Infatti, d'accordo con il fratello Gian Battista, propagò fra il clero e fra i cittadini le nuove dottrine, diffondendo manoscritti, lettere, rinnegando le confessioni dei peccati, ecc. Solo cinque anni più tardi Pier Paolo venne spogliato della dignità episcopale.'

Al proliferare della riforma si era opposto anche papa Paolo IV, ma a livello istriano il vero grande aiuto alla curia romana fu dato dall'opera svolta da Girolamo Muzio, che in seguito alla pubblicazione delle Vergeriane e a causa della polemica con il Vergerio, fu ritenuto il vero salvatore del cattolicesimo in Istria. Le Vergeriane rappresentano il capolavoro dell'epoca per quel che riguarda la storia ecclesiastica della diocesi di Capodistria. $^{\text {Io }}$

\section{Muzio e la Controriforma}

Girolamo Muzio ebbe il suo primo approccio con il luteranesimo nel 1545, quando accompagnò il marchese del Vasto alla corte di Carlo V, che allora si trovava a Worms in Germania. S'informò sulla dottrina e i costumi degli innovatori e si convinse che le dottrine protestanti fossero eretiche e che fosse suo obbligo impedire la diffusione della riforma in Italia. ${ }^{\text {II }}$

In un gruppo di lettere che appartengono a un periodo poco conosciuto della vita del Muzio fra il 1553 ed il 1558 , conservate fra le carte del cardinale Gian Pietro Carafa, membro dell'Inquisizione, appare evidente che il Muzio agiva come spia dell'Inquisizione e anche come agente provocatore, assumendo alle volte pure un atteggiamento di superiorità, tendendo a esagerare, senza cattiveria, ma per ambizione, i mali o le colpe nelle quali s'imbatteva. ${ }^{\mathrm{I}}$

\footnotetext{
9 Miculian, "Il Santo Ufficio e la riforma protestante in Istria," 215 230.

Io Miculian, "Il Santo Ufficio e la riforma protestante in Istria," I73230.

I I Miculian, "Il Santo Ufficio e la riforma protestante in Istria," I73230.

I2 Miculian, "Il Santo Ufficio e la riforma protestante in Istria," I73230.
} 
Tra queste lettere sono interessanti quelle indirizzate ad alcuni concittadini di Capodistria, e si potrebbero classificare come un'appendice alle Vergeriane. Da esse sappiamo che nel I533 alcuni dotti cittadini di Capodistria si erano uniti per formare un'accademia, come tante che si fondavano in Italia, con il nome di accademici desiosi, decidendo che non si doveva menzionare la religione per impedire qualsiasi contesa o disputa. Naturalmente Muzio interpretò nel peggiore dei modi la loro decisione e subito scrisse, da Venezia, la sua prima lettera agli accademici che considerarono l'intervento inutile e scandaloso. ${ }^{13}$

L'autore spesso, nella morale e nelle controversie teologiche, si dimostrò contraddittorio con se stesso. Nei suoi scritti più volte criticò, detestando quello che pure lui stesso praticava. Ad esempio, nel Trattato della comunione de' laici e delle mogli de' chierici (pubblicato con le Vergeriane), lodò e sostenne la continenza, seppur anche lui viveva in concubinato e fu amante di Tullia D'Aragona. ${ }^{14}$

Nel 1533 pubblicò i Tre testimonifedeli, dedicato alla duchessa Vittoria Farnese, dove mostrò quali erano le dottrine nelle chiese d'Europa, d'Africa e d'Asia nel secondo, terzo e quarto secolo dopo Cristo, spiegando le frodi d'Erasmo. ${ }^{\text {Is }}$

Sostenne, invece, l'autorità del concilio con varie opere. Scrisse il Bulligero riprovato (I562), la De Romana Ecclesia (1563), l'Eretico infuriato (I562), la Cattolica disciplina de' Principi (I562), l'Antidoto cristiano ( 1562 ), che parla delle menzogne che si trovano nelle prediche di Bernardino Ochino. Contro di lui aveva scritto anche le Mentite ochiniane (155I). ${ }^{16}$

Quando morì papa Pio IV nel 1566, Muzio si affidò a un altro potente protettore, papa Pio $\mathrm{V}$

\footnotetext{
I3 Pio Paschini, "Episodi della Controriforma in lettere inedite di Girolamo Muzio," in Atti e Memorie della società istiana di archeologia e storia patria, Vol. XXXIX-fascicolo I (Trieste: Società istriana di archeologia e storia patria, 1927), 349-350.

I4 Pietro Stancovich, "Biografia degli uomini distinti dell'Istria," in Atti del Centro di Ricerche Storiche, Vol.3 (Rovigno-Trieste: Centro di Ricerche Storiche di Rovigno, 1972).

is Paolo Giaxich, Vita di Girolamo Muzio (Trieste: J. Papasch\&Tip., I847), 52 .

I6 Giaxich, Vita di Girolamo Muzio, 58-59
}

che gli concesse una grossa provvigione. Nel 1570 scrisse l'Istoria Sacra, nella quale lotta contro gli errori e le frodi con cui gli eretici cercavano di offuscare le verità dei Vangeli. Scrisse inoltre: Difesa della messa, de' santi, del papato, contro le bestemmie di Pietro Viretro (1565), la Beata Vergine incoronata (1567), il Coro pontificale (1570), Lettere cattoliche (1571), la Selva odorifera (1572). ${ }^{17}$

\section{La questione della lingua italiana}

Nei primi decenni del XVI secolo, abbandonata l'idea umanistica di usare il latino come lingua letteraria, iniziarono accesi dibattiti intorno alla lingua che miravano alla ricerca e alla determinazione del tipo di lingua letteraria che avrebbero dovuto usare tutti gli scrittori d'Italia e a fissare le regole grammaticali. ${ }^{18}$

Già Dante, nel De vulgari eloquentia si era posto l'impegno di indicare agli scrittori in volgare un modello di lingua italiana, valido per tutti gli autori, al di là della frammentazione geografica e politico-culturale che presentava già da allora l'Italia. Tale modello doveva essere una koinè linguistica e cioè una lingua comune in cui venivano a mescolarsi i diversi volgari italiani nei loro aspetti più "illustri” e cioè più nobili, depurati dai più evidenti elementi di rozzezza plebea, sia in campo lessicale (il vocabolario) sia morfologico e sintattico. Dante è stato perciò il primo grande scrittore italiano a prefigurare una lingua unitaria di estensione "nazionale". Tuttavia, è evidente il fatto che, nella concretezza della sua scrittura, soprattutto nella "Commedia", Dante rimane in gran parte fedele al fondo toscano-fiorentino della lingua materna e il "volgare illustre" resta un modello ideale astratto, lontano da ogni possibilità di realizzazione concreta. ${ }^{\mathrm{I}}$

A cominciare dal Petrarca che, come affermano alcuni critici, esercitò una dittatura linguistica involontaria attraverso i secoli successivi, si afferma sempre più il modello del fiorentino illustre e si apre, a partire dal 'soo, un dibattito

\footnotetext{
I7 Giaxich, Vita di Girolamo Muzio, 58-59.

I8 Alberto Asor Rosa, Sintesi di storia della letteratura italiana (Firenze: La Nuova Italia, 1978), 98-99.

19 Nives Zudič Antonič, Storia e Antologia della letteratura italiana di Capodistria, Isola e Pirano (Capodistria: Unione italiana, 2014), 86.
} 
a più voci sulla lingua italiana, parlata e scritta, che si spinge fino al nostro tempo. ${ }^{20}$

Per tutto il Cinquecento la "questione della lingua" occupa un posto centrale. Si tratta soprattutto di proporre un codice linguistico per gli scrittori, un modello cioè di lingua scritta (non parlata) nobile e adatto a temi elevati quali l'amore spirituale, la fede religiosa, le gesta eroiche, ecc. Il più importante in tal senso è: "Le prose della volgar lingua" del veneziano Pietro Bembo. Nell'opera, tre libri in forma di dialogo (genere prediletto dai trattatisti del 'soo), si sostiene la seguente tesi: la lingua degli scrittori (a differenza di quella di Dante che appare al Bembo troppo carica di forme plebee, di rozzezze, di neologismi astrusi) doveva basarsi sul fiorentino colto: quello usato dal Petrarca per la poesia e da Boccaccio per la prosa. ${ }^{21}$

La tesi del Bembo incontrò subito forti opposizioni. Ne erano contrari soprattutto coloro che pur considerando l'importanza del fiorentino, non ne accettavano la preminenza e preferivano una lingua più variegata che prendesse spunto dalle forme delle varie parlate d'Italia. Si trattava infatti del ritorno alla tesi dantesca, cioè a quella della koinè, ad essa si rifaceva Baldassar Castiglione, con la sua opera il Cortegiano, e Giangiorgio Trissino, il principale avversario del Bembo, veneto pure lui, il quale, nel I529, pubblicò un dialogo, il Castellano, in cui sostanzialmente ripropone la teoria dantesca. Attorno a queste due tesi, da una parte il "fiorentinismo bembesco" e dall'altra la "lingua italiana" del Trissino, si raccolsero tutti gli altri letterati che intervennero nel dibattito sulla lingua da posizioni diverse. Chi volendo inserire nel modello bembesco anche termini tratti dalla lingua fiorentina parlata, chi affermando che la lingua da usare avrebbe dovuto essere quella toscana. Alla fine però vinse la tesi del Bembo, e la lingua italiana letteraria, fino alla metà del Settecento, era quella basata sul modello dei due grandi letterati

20 Zudič Antonič, Storia e Antologia della letteratura italiana di Capodistria, Isola e Pirano, 86.

2 I Zudič Antonič, Storia e Antologia della letteratura italiana di Capodistria, Isola e Pirano, 86 del Trecento e andava studiata attraverso i libri e la tradizione letteraria. ${ }^{22}$

\section{Girolamo Muzio e la questione della lingua}

Anche nella nostra regione, molti letterati affrontarono la questione della lingua italiana, in particolar modo tra di loro si distinse Girolamo Muzio che studiò le teorie bembesche ed era suo fermo sostenitore.

Muzio iniziò probabilmente ad occuparsi della questione della lingua durante un suo viaggio in Dalmazia e precisamente sull'isola d'Arbe dove si recò nel I5I3. Nell' isola dalmata d'Arbe, Muzio discuteva con Antonio Mezzabarba, discreto poeta veneziano e buon raccoglitore di rime antiche, delle regole della lingua italiana e gli fece probabilmente conoscere le teorie di Bembo. Inoltre, quando si trovò al servizio di Pietro Bonomo, ebbe l'opportunità di entrare in contatto e leggere un libro di grammatica del dalmata Francesco Fortunio, ${ }^{23}$ e da Trieste dove si trovava al servizio del vescovo Pietro Bonomo, Muzio ne scriveva con grande interesse ad Aurelio Vergerio:

\section{È stato mandato al vescovo mio un libro di grammatica volgare, di un messer Francesco dalmatino; la quale a me è stata cara di vede- re, perché egli insegna di molte cose, e della ortografia, e della proprietà della lingua, del- la variazion de’ nomi e de’ verbi.[.... E dob- biam sperar di vedere che la lingua in questo non si fermerà, che' ne avremo anche degli altri scrittori. ${ }^{24}$}

Più tardi intervenne al dibattito con vari scritti linguistici composti tra il 1533 e il 1574 e

22 Nives Zudič Antonič. "Girolamo Muzio e la questione della lingua italiana," in Hieronimo Mutio Iustinopolitano: Operette morali (Giolito, in Vinegia 1550), Le Vergeriane (Giolito, in Vinegia I550), Egloghe (Giolito, in Vinegia 1550), Le battaglie (Dusinelli, in Vinegia I582), Il duello (Compagnia de gli Uniti, in Venetia 1585), Lettere (Sermartelli, in Firenze 1590), cur. Petre Štoka e Ivan Markovič (Capodistria: Biblioteca centrale Srečko Vilhar, 2013), 79-93.

23 La grammatica di Francesco Fortunio Regole grammaticali della volgarlingua (I516) era considerata la prima grammatica italiana a stampa.

24 Girolamo Muzio, Lettere inedite di Girolamo Muzio Giustinopolitano: pubblicate nel IV centenario della sua nascita (Capodistria: Comune, 1846), i I. 
pubblicati postumi dal figlio Giulio Cesare con il titolo di Battaglie in difesa dell'italica lingua (I582). ${ }^{25}$

Nel 1530 Muzio si recò a Parigi con il conte Rangone. In questa città conobbe Luigi Alamanni con cui poté affrontare il tema che gli stava particolarmente a cuore: l'uso della lingua italiana.

Nel 1535 si recò a Ferrara, dove ebbe l'opportunità di conoscere due letterati fiorentini, Bartolomeo Cavalcanti e Gabriele Cesano e le loro opinioni sulla lingua. I due letterati fiorentini erano grandi ammiratori dello stile di Machiavelli e criticavano quello di Boccaccio. Muzio affermò il contrario e nella sua opera le Battaglie, riconobbe ogni grazia dello stile al Boccaccio. ${ }^{2}$

E per tornare al Boccaccio, istimo io che alcuno non viva oggi, che in questa lingua scriva in prosa con alcuna lode, che da altro volgare scrittore che da lui abbia imparato a scrivere. E se di me mi fosse lecito alcuna cosa dire, io direi che io ho pure alcuna volta fatto prova di scrivere alcuna cosa: ho scritto di molte e di varie lettere; ho scritto in suggetto di duello; ho scritto alcuna cosa morale; et a materie di più dignità sono ancora trapassato. Né ho io alcuna cosa scritto con alcuna diligenza, che io scritta non l'abbia in quella lingua che dalle novelle del Boccaccio ho imparata. Né infino ad ora mi pento di averla imparata tale. Non mi siano queste parole di me apposte a vizio, che io per altro a dirle non mi sono condutto, se non per argomento: che io tale dalla imitazione del Boccaccio in tante maniere di dire mi sono avanzato, non si doverà negare che gli uomini di rara dottrina e di chiaro ingegno non siano per trattar con quella ogni altissimo suggetto. $^{27}$

25 "Il Muzio grammatico, secondo un libro recente," Pagine istriane n.6 (1909): 132-133.

26 Nives Zudič Antonič, Storia e Antologia della letteratura italiana di Capodistria, Isola e Pirano, 98.

27 Girolamo Muzio, Battaglie di Hieronimo Mutio giustinopolitano, per diffesa dell'italica lingua, con alcune lettere a gl'infrascritti nobili spiriti (Venezia, 1582), ir.
Il Muzio ammira lo stile di Boccaccio e non apprezza quello del Machiavelli, a differenza di quanto affermano messer Gabriello e messer Cesano che dicono che Machiavelli sia più dotato di grazia e di eleganza. Secondo il Muzio invece il Machiavelli non scrive bene e lui pertanto non ha mai preso spunto dalle sue opere.

E dico che de' libri del Macchiavelli già è gran tempo che me ne vennero alcuni in mano, et avendone nella lezion di poche righe il suo stilo e la sua lingua notata, gli gittai da parte, come quegli da' quali io non pensava di poter raccogliere cosa di tanta utilità di quanto danno potrebbe essere stato quel suo dire alle mie scritture. [...] Io non so trovar nelle parole di lui cosa che comportabile mi paia in scrittore che voglia con lode alcuna cosa scrivere. Se riguardo alla forma del dire, non so come dir si possa più bassamente. Se cerco degli ornamenti, non ne trovo niuno: anzi mi pare egli esser tutto secco, e digiuno di ogni leggiadria. Poi nella lingua egli è tale, che oltra l'usar molte parole latine, là dove non men belle ne averebbe avute delle volgari, e nella variazione e nella proprietà de' verbi egli è tutto cieco; usa male i nomi, e peggio i pronomi; non sa ben collocare né articoli né adverbii: et in somma tanto sa delle osservazioni della lingua, quanto chi non ne sa niente. ${ }^{28}$

-

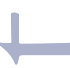

Le Battaglie in difesa dell'italica lingua, è l'opera di Muzio che, escludendo il settore religioso, poetico e cavalleresco, si può oggi ritenere quella di maggiore rilevanza culturale.

L’opera, concepita forse già nel I530, fu pubblicata solo nel 1582 , accompagnata inoltre da lettere indirizzate ai maggiori esponenti che si occuparono della questione della lingua. ${ }^{29}$

Secondo Bonora, in questi scritti Muzio diede prova migliore del suo acume, sostenendo l'italianità del volgare, rifacendosi ad una lingua colta, da apprendere attraverso lo studio severo

28 Muzio, Battaglie di Hieronimo Mutio giustinopolitano, per diffesa dell'italica lingua, con alcune lettere a gl'infrascritti nobili spiriti, $4 \mathrm{r}$.

29 Aldo Cherini e Paolo Grio, Le famiglie di Capodistria: notizie storiche ed araldiche (Trieste: Famea Capodistriana, 1998), 17ı. 
degli antichi scrittori. Riconosce, inoltre, l'importanza degli scrittori moderni d'origine non toscana, in particolare dell'Ariosto e persino degli scrittori dialettali. ${ }^{30}$

\section{Come nasce la lingua letteraria}

Per quanto riguarda la questione della lingua, come abbiamo già affermato, Muzio si rifà alle teorie del Bembo, ed è concorde con il fatto che ogni lingua ha una sua parabola di sviluppo, salendo via via alla sua perfezione. Pertanto anche la lingua italiana è destinata ad una tale ascesa verso la sua perfezione espressiva e gli scrittori devono promuovere tale svolgimento rifacendosi alla più alta tradizione del passato. Perciò devono prendere a modello il Petrarca e il Boccaccio, che hanno dimostrato con le loro opere il grande valore del volgare italiano e cercare di perfezionare la loro lingua.

Nelle lettere e soprattutto nella raccolta che prende il nome di Battaglie in difesa dell'italica lingua Muzio sostiene la teoria fondamentale di Bembo, e precisamente che la lingua letteraria non nasce o si governa secondo l'uso dei parlanti, ma si sviluppa e deriva da una tradizione letteraria, cioè mediante l'opera degli scrittori. Inoltre spiega che non è importante essere nati a Firenze $\mathrm{o}$ in Toscana per poter scrivere bene, ma che per scrivere bene bisogna imparare dai libri. Secondo Muzio per apprendere bene una lingua bisogna studiarla attentamente attraverso la lettura di grandi opere e fare sempre attenzione alla propria scrittura. Pertanto, secondo lui, anche coloro che non sono nati a Firenze o in Toscana, impegnandosi, possono scrivere bene. ${ }^{32}$

Enel vero le lingue, le vere lingue, non si imparano dalla mamma, e non dal babbo, ma dalle scritture: e là dove voi altri volete (voi dicendo intendo di dire a coloro che così sentono) che dal parlare a scrivere si impa-

30 Ettore Bonora, "Il classicismo dal Bembo al Guarini," in Storia della Letteratura Italiana, vol. IV, Il Cinquecento (Milano: Garzanti, 1966), 184 .

$31 \quad$ Nives Zudič Antonič. "Girolamo Muzio e la questione della lingua italiana," 83

32 Nives Zudič Antonič. "Girolamo Muzio e la questione della lingua italiana," 83. ri, ho io da Cicerone imparato che dal leggere e dallo scrivere si impara a parlare. Ma per meglio intendere qual via si debbia tenere per conseguir dirittamente questa più nuova lingua, avviso che sia ben fatto che dalle altre più antiche se ne debbia prendere l'essempio. Epertanto, se volete conoscere quanto poco giovi l'esser nato toscano per volere toscanamente scrivere, ricordivi che Virgilio fu mantovano, Catullo veronese, Orazio venusino, Terenzio africano, e che Marco Tullio non fu romano. Da cui vogliam noi dire che quegli stranieri apprendessero la romana lin gua? Da' libri $[\ldots]^{33}$

Muzio prosegue dando una descrizione di se stesso e del suo scrivere in italiano, dicendo che gli studiosi fiorentini lo ritenevano incapace di scrivere bene perché non era nato a Firenze. ${ }^{34}$

E prima dico che io sono per origine della città di Iustinopoli, volgarmente detta Capodistria, e dagli antichi appellata Egida, lontana dal Carnaro, Ch'Italia chiude, e suoi termini bagna, intorno ad ottanta miglia. Nacqui in Padova e fra in Padova, in Vinegia, in Capodistria, in Dalmazia et in Alamagna vissi in fino alla età di trenta anni. Appresso conversai in Lombardia, in Piemonte, in Francia, et in Fiandra; e ne aveva forse quaranta prima che Fiorenza mi vedesse, et a mettere insieme tutto il tempo che in più volte stato vi sono, non so se egli passasse un anno. Sì che né io vi son nato, né da fanciullo allevato: e che in me non sia indicio alcuno di fiorentina ria, assai si mostra a chi mi sente favellare. Laonde per la coloro ragione si viene a conchiudere che io bene non posso scrivere. ${ }^{35}$

Lo scrittore prosegue poi dicendo che anche gli scrittori istriani, come quelli fiorentini, hanno appreso la lingua italiana dalle loro madri e si fanno capire da tutti coloro che parlano italiano,

33 Muzio, Battaglie di Hieronimo Mutio giustinopolitano, per diffesa dell' 'italica lingua, con alcune lettere a gl' infrascritti nobili spiriti, $4 \mathrm{r}$.

34 Nives Zudič Antonič. "Girolamo Muzio e la questione della lingua italiana," 87

35 Muzio, Battaglie di Hieronimo Mutio giustinopolitano, per diffesa dell'italica lingua, con alcune lettere a gl' infrascritti nobili spiriti, $34 \mathrm{r}$. 
pur non usando la pronuncia fiorentina; inoltre Muzio afferma che gli scrittori istriani e lui pure, hanno migliorato la loro conoscenza della lingua italiana attraverso i libri e lo fanno meglio dei fiorentini che pensano di sapere perfettamente la lingua e di non avere bisogno di migliorarla. ${ }^{36}$

Abbiamo anche noi succhiata la lingua italiana dalle poppe delle balie e delle madri, e dal popolo e da' cittadini delle nostre città la abbiamo appresa: e con questa nostra lingua, et io e degli altri andiamo per tutta Italia parlandola. Et io, uno fra gli altri, dal Varo all'Arsa la ho corsa tutta, e per tutto sono stato inteso: e si mi sentito parlare, e se da loro è stata intesa la mia favella, quantunque ella non mi gorgogliasse nella strozza alla fiorentina. Da' libri ci vantiamo noi di apprendere a 'dirittamente scrivere'. [...] Da' libri impariamo noi a bene scrivere, e più agevolmente impariamo noi che i Fiorentini, né gli altri Toscani, perciò che come noi ci mettiamo a voler dar opera allo studio dello scrivere, così ci persuadiamo di non saperne nulla; e perciò negli animi nostri, come in tavole monde, si figurano le bellezze e le purità della lingua, in chi vuol faticare, e sa studiare; e questi sono molto pochi. ${ }^{37}$

Muzio si occupò nell'arco dell'intera vita della questione della lingua, e soprattutto si adoperò in difesa di chi, non essendo nato a Firenze, poteva dimostrare come lui di essere un buon conoscitore della lingua italiana, grazie alla lettura e al costante impegno. Albino Zenatti, invitato dal municipio di Capodistria a pubblicare alcune lettere inedite di Girolamo Muzio nel quarto Congresso della lega nazionale, in occasione del quarto centenario della sua nascita disse di lui le seguenti parole: "Fra i molti letterati che illustrarono Capodistria, nessuno, in vero, si occupò de-

36 Nives Zudič Antonič. "Girolamo Muzio e la questione della lingua italiana," 87.

37 Muzio, Battaglie di Hieronimo Mutio giustinopolitano, per diffesa dell' italica lingua, con alcune lettere a gl' infrascritti nobili spiriti, 39r.

gli studi intorno alla nostra lingua più o meglio del Muzio". ${ }^{8}$

\section{Le opere di Girolamo Muzio \\ Il Duello}

Il trattato Duello di Girolamo Muzio fu stampato per la prima volta a Venezia nel I550, assieme alle Risposte cavalleresche e immediatamente ebbe un enorme successo editoriale non solo in Italia, ma in tutta Europa. Con quest'opera, divenne l'autore di scienza cavalleresca più noto del secolo.

Il suo testo fu considerato un'opera innovativa, perché scritta in volgare, da un letterato o meglio da un cortigiano e non da un uomo di legge o d'armi, suscitando tra il pubblico disinteresse per i precedenti trattati. ${ }^{39}$

Il Muzio fu "duellista" e "duellante", secondo il senso delle voci stabilito all'epoca da un altro trattatista de Il Duello, Giovambattista Pigna:

"Duellista sarà colui che scriverà di duello: il quale fondandosi su questo suggetto e da esso essendo nominato, farà il duello essere scienza. Duellante [...] chiunque entri in duello: e da questa voce, come da quella che è suo mestiero, il nome piglierà" ${ }^{40}$

"Duellante", divenne, seppur "in punta di penna" nel momento in cui si apprestò ad assumere - per conto d'Alfonso d'Avalos Marchese del Vasto, luogotenente imperiale a Milano, l'ufficio di "duellista". ${ }^{41}$

Ben presto in molti si affidarono alle sue consulenze per risolvere le diverse querele in "suggetto di honore". L'autore stesso le menzionò nella sua opera le Vergeriane. Inoltre le diverse consulenze furono pubblicate nell'opera le Risposte cavalleresche.

38 Albino Zenatti, Lettere inedite di Girolamo Muzio Giustinopolitano: pubblicate nel IV centenario della sua nascita (Capodistria: Comune, I846), 5 .

39 Francesco Erspamer, La biblioteca di Don Ferrante. Duello e onore nella cultura del Cinquecento (Roma: Bulzoni, 1982), 82.

Giovambattista Pigna, Il duello (Venezia: Valgrisi, I554), I45.

$4^{1} \quad$ Luciana Borsetto. "L'Ufficio di scrivere in suggetto di honore'. Girolamo Muzio 'duellante' duellista'," Acta Histriae IX (2000): $14 \mathrm{I}$. 
IlDuello era diventato "la soluzione finale di tutte le controversie insorte 'per cagion di honore”. Le sue furono semplici opinioni e pareri su argomenti che gli venivano sottoposti. ${ }^{22}$ Per scrivere la sua opera, quindi, non dovette far altro che mettere per iscritto il suo sapere specialistico che aveva acquisito, in quanto uomo di corte, e che aveva approfondito con le proprie ricerche personali. È Muzio stesso a dichiararlo nella dedicatoria a Emanuele Filiberto:

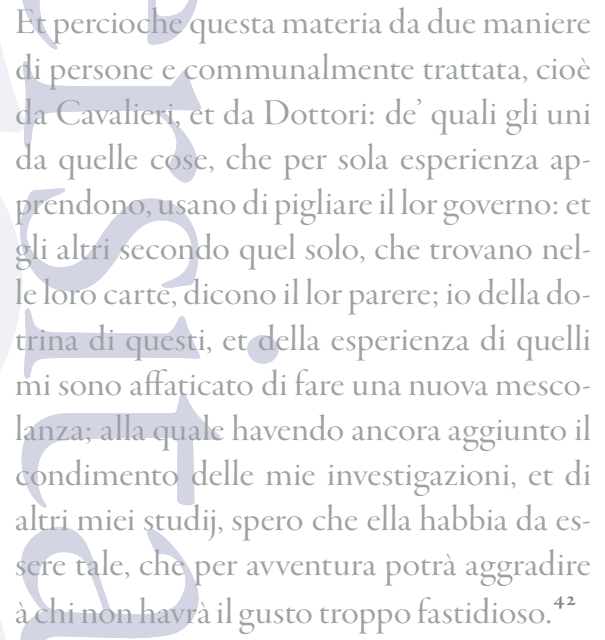

L'autore duellò con la penna contro coloro che non rispettavano le regole cavalleresche, che non rispettavano la fede cristiana, in nome della lingua italiana e in nome del papato. ${ }^{43}$

Con quest'opera voleva porgere ai cavalieri uno strumento efficace affinché potessero con onore districare le questioni cavalleresche. Il trattato presentava il duello come un cerimoniale di norme, di consuetudini allo scopo di disciplinarne l'uso. Il suo intento era nobile; considerando che sarebbe stato impossibile abolirlo, voleva almeno diminuirne l'uso e soprattutto impedirne l'abuso. $^{44}$

Secondo l'autore, il duello, non doveva venir praticato per sopraffazione o vendetta, ma come

42 Girolamo Muzio, Il duello del Mutio Iustinopolitano, con le risposte cavalleresche (Venetia: Compagnia degli Uniti, I585), 3.

43 Borsetto, "L'Ufficio di scrivere 'in suggetto di honore'. Girolamo Muzio 'duellante' duellista'," I4I.

44 Giaxich, Vita di Girolamo Muzio, 47. Erspamer, La biblioteca di Don Ferrante. Duello e onore nella cultura del Cinquecento, I 12. "prova della verità" nonché come legittima difesa nei "confronti dell' ingiuria subita". 45

L'obiettivo dell'autore era duplice: “descrivere l'arbitrio che governava a suo tempo la Pratica perversa del combattimento privato dei nuovi cavalieri, dibattuti tra ingiuria e 'mentita', tra infamia e onore, tra offesa e vendetta, volti principalmente al conseguimento della comune approvazione, e sottoporlo al vero costume della cavalleria”. Nella dedicatoria a Emanuele Filiberto leggiamo:

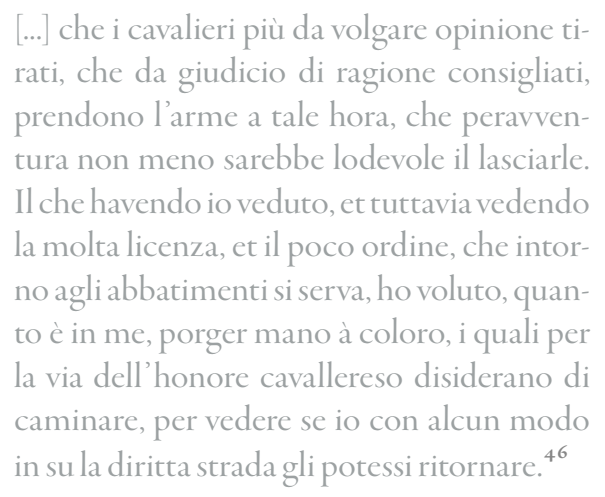

S'impegnò anche a proporre denominazioni specifiche, importando alcuni termini come "attore", "reo", dicendo con orgoglio di essere stato il primo, a parlare di "mentite". Gli scrittori usavano in generale un lessico specifico, affatto originale, destinato ad un pubblico eterogeneo, quindi doveva venir compreso pure da persone poco colte. $^{47}$

Muzio trattò d'ingiurie, delle mentite, dell'attore, del reo, dei cartelli, del campo, esplicando i loro significati per una corretta utilizzazione. Dichiarò che per vendetta i signori non dovevano concedere il campo, spiegò come si arrivava al duello "per delitto" e teorizzò su come doveva essere il "risentimento onorevole" e a "quali gradi della nobiltà" era consentito combattere. Stabilì chi era il "reo" e chi l'“attore", come si configurava l'“ingiuria” e come il "ca-

\footnotetext{
45 Borsetto, "L'Ufficio di scrivere 'in suggetto di honore'. Girolamo Muzio 'duellante' 'duellista," I 4 I.

Muzio, Il duello del Mutio Iustinopolitano, con le risposte cavalleresche, 3 .

47 Erspamer, La biblioteca di Don Ferrante. Duello e onore nella cultura del Cinquecento, 130-136; Borsetto, "L'Ufficio di scrivere 'in suggetto di honore'. Girolamo Muzio 'duellante' 'duellista'," 147.
} 
rico", sotto che titoli si dovevano congegnare le "mentite", sotto che forma dovevano venir concesse le "patenti di campo" e quali armi usare negli "steccati". Muzio, così, riformò il moderno duello. "Notando, et elevando" gli abusi che lo rendevano crimine, sottoponendolo a nuove leggi. $^{48}$

In realtà, quanto si può dedurre dalle missive che Muzio scrisse ad amici e nemici, pare che iniziò a scrivere l'opera, soprattutto perché sollecitato dai propri signori e padroni, ovvero gli fu imposto. Ciò è quanto traspare nella dedicatoria:

$$
\begin{aligned}
& {[\ldots .] \text { à prender questa honorevole fatica non }} \\
& \text { picciolo sprone mi è stato (illus. Prencipe) } \\
& \text { l'havere io veduto quanto il gentilissimo } \\
& \text { amico vostro fosse desideroso, che io alcu- } \\
& \text { na cosa scrivessi in questo suggetto (che nel } \\
& \text { tempo, nel quale io mandato dal mio Signor } \\
& \text { Marchese a Nizza di Provenza àil servire il S. } \\
& \text { Duca vostro padre, et voi, non una sola volta } \\
& \text { da voi imposto mi fu, che dovendovi io man- } \\
& \text { dare delle mie scritture, ve ne mandassi in } \\
& \text { materia di Duello). Per tal cagione già vi ap- } \\
& \text { prestai io, et hora ho pubblicati questi miei } \\
& \text { libri, giudicando che le cose scritte in sug- } \\
& \text { getto di onore ad honorato Prencipe otti- } \\
& \text { mamente si convengono. }{ }^{49}
\end{aligned}
$$

Per rispondere alle polemiche che il suo libro aveva provocato, progettò inoltre di realizzare un libro particolare di Questioni di Duello, proponendosi di rispondere a coloro che si "erano dilettati di trafiggerlo", ma la censura del 1563 ne bloccò la realizzazione. Il suo nuovo testo seguì le sorti che erano state riservate anche ad altri trattati inerenti allo stesso tema, tuttavia il trattato Il Duello, fino al 1585 , continuò ad essere pubblicato. ${ }^{\text {so }}$

I censori però impedirono all'autore di portare a termine l'opera con cui avrebbe risposto a

\footnotetext{
48 Borsetto, "L'Ufficio di scrivere 'in suggetto di honore'. Girolamo Muzio 'duellante' duellista', I48.

49 Borsetto, "L'Ufficio di scrivere 'in suggetto di honore'. Girolamo Muzio 'duellante' "duellista', I 4 8-I 49.

50 Erspamer, La biblioteca di Don Ferrante. Duello e onore nella cultura del Cinquecento, 63.
}

tutti coloro che lo avevano attaccato, accusandolo "di aver discettato su tutto, di essersi occupato di vili soggetti e indegni di esser messi in scrittura, di aver condotto gli uomini a morte, di aver voluto trattare, da 'huomo secolare, et di corte', materie pertinenti alla cattolica disciplina; di aver desunto il suo sapere dalla scienza degli altri, usurpando una fama immeritata".

"Allineate su un unico piano, l'icona del Muzio 'duellante' che ci rinviano le sue opere nell'ultimo scorcio del secolo, si sovrappone del tutto a quella del Muzio 'duellista', emblemi entrambe dell'unica, ininterrotta fatica da lui compiuta nel suo ossequio ai poteri secolari e cattolici del tempo: l'“ufficio di scrivere in suggetto di honore"'.52

La sua fama di "duellante" terminò con la sua morte nel 1576 , quella di scrittore invece no. Infatti, Manzoni lo cita nella sua opera I Promessi Sposi, inserendolo nella biblioteca del personaggio di don Ferrante. ${ }^{53}$

\section{Le lettere}

In generale la lettera del Cinquecento (in particolare quella del Muzio) non fu vincolata da nessuna necessità di tempo o di luogo, ma al tempo indeterminato della comunicazione letteraria. Muzio fece ampio uso di questo strumento di comunicazione. Il suo "luogo" fu quello soggettivo della lettura e della rilettura, tanto che lui stesso incitava i lettori a leggere e a rileggere una stessa lettera, in modo da farla diventare lunga a piacere, quanto vorremmo noi. ${ }^{54}$

Nella dedicatoria a Vincenzo Fedeli delle Lettere (denominate dall'autore secolari, per distinguerle dalle cattoliche) in tre libri (il quarto venne pubblicato nel 1590 , dopo la morte dell'au-

\footnotetext{
5I Borsetto, "L’Ufficio di scrivere 'in suggetto di honore'. Girolamo Muzio 'duellante' 'duellista', I5 1 .

52 Borsetto, "L'Ufficio di scrivere 'in suggetto di honore'. Girolamo Muzio 'duellante' 'duellista," I55

53 Alessandro Manzoni, I Promessi Sposi (Milano: Principato, 1976), Capitolo XXVII, 496

54 Luciana Borsetto, "Introduzione," in Muzio Girolamo: Lettere. Rist.anastat. (Bologna: Forni, 1985), XIII.

$\checkmark$

$\sqrt{n}$

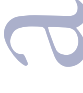

$\sqrt{ }$


tore), è palese la nozione fondamentale da cui traeva origine l'intera raccolta. ${ }^{55}$

\section{Certissima cosa è, che lo scrivere delle lette- ré non per altro è stato intodutto, se non per mantenener viva la humana conversatione, la quale dalla lontananza essendo interrotta, senza quelle se ne verrebbe del tutto à mori- re. [...] le lettere sono esse le mantenitrici del- la conversazione $[\ldots]^{56}$}

Le lettere del Muzio dimostrano il suo sapere su questioni largamente dibattute nelle corti di quel periodo: l'amore, l'astrologia e in particolare la lingua. Quelle di Muzio possono essere anche argomentazioni morali e didattiche, o semplicemente digressioni su luoghi o su personaggi. Tutto ciò ci riporta alla figura dell'erudito e dell'intellettuale del Cinquecento, in grado di discutere e di approfondire i più disparati discorsi. Le lettere servirono però a Muzio come veicolo privilegiato dei suoi vari duelli su carta di cortigiano-scrittore, per condannare o per assolvere, per ricusare o per smentire, per difendere gli altri o se stesso. ${ }^{57}$

Le Vergeriane e le Catholiche possono essere qualificate come vere e proprie dispute a distanza, composte allo scopo di smascherare coloro che si dimostrarono contrari a tutto ciò che esse contenevano. ${ }^{58}$

Volendo fare una ripartizione delle lettere di Muzio va detto che all'inizio i temi trattati furono l'amore e l'amicizia mentre in seguito discusse temi più specifici come la lingua, l'onore, la cavalleria e le armi in particolare. Durante il Concilio di Trento, invece, diventarono lettere istruttorie e didattiche sulla formazione del buon principe cattolico. In questo modo, però, si persero le funzioni vere e proprie di questo modello letterario, seguendo la linea dell'argomentazione tipica del trattato. Sono in forma più

\footnotetext{
55 Borsetto, "Introduzione," IX.

56 Muzio, Il duello del Mutio Iustinopolitano, con le risposte cavalleresche, 1585 .

57 Borsetto, "Introduzione," XIX; Borsetto, "L'Ufficio di scrivere 'in suggetto di honore'. Girolamo Muzio 'duellante" duellista,", 152.

Borsetto, "Introduzione," LV
}

58 lunga, così l'autore si vede costretto a normalizzare l'eccessiva lunghezza. ${ }^{59}$

La forma epistolare, quasi universalmente utilizzata dal Muzio, diventò come già detto la "forma stessa del suo scrivere", utilizzata anche nelle Risposte cavalleresche, pubblicate separatamente o a seguito del Duello, e in alcuni scritti, pubblicati sotto il titolo di Operette morali e di Avvertimenti morali. Per ultimo, ma non per importanza, da ricordare ancora un'altra raccolta dal titolo: Battaglie in difesa dell' italica lingua. ${ }^{60}$ Lettere inedite

Il municipio di Capodistria invitò Albino Zenatti a pubblicare alcune lettere inedite di Girolamo Muzio nel quarto Congresso della lega nazionale, in occasione del quarto centenario della nascita di quel "valoroso letterato", che nacque a Padova. Il volume è un' interessante fonte per approfondire la vita e la personalità di Girolamo Muzio.

Come dice Zenatti: "Fra i molti letterati che illustrarono Capodistria, nessuno, in vero, si occupò degli studi intorno alla nostra lingua più o meglio del Muzio". ${ }^{61}$ Dalle regioni del Veneto, nei primi anni del Cinquecento partirono le teorie che fecero definitivamente trionfare il volgare sul latino, e la lingua di Dante e Petrarca divenne veramente lingua letteraria della nazione intera. Sicuramente il merito principale del movimento cinquecentesco a favore del volgare, spetta al veneziano Pietro Bembo.

Nell' isola dalmata d'Arbe, il Muzio discuteva con Antonio Mezzabarba, discreto poeta veneziano e buon raccoglitore di rime antiche, delle regole della lingua italiana. Era stata da poco pubblicata l'operetta grammaticale di Francesco Fortunio e da Trieste dove si trovava al servizio del vescovo Pietro Bonomo, Muzio ne scriveva con grande interesse ad Aurelio Vergerio:

\footnotetext{
È stato mandato al vescovo mio un libro di grammatica volgare, di un messer Francesco

Borsetto, "Introduzione," LV.

60 Per quanto riguarda la lingua Muzio favori la tesi di Dante e Trissino. Borsetto, "Introduzione," LV.

6I Zenatti, Lettere inedite di Girolamo Muzio Giustinopolitano: pubblicate nel IV centenario della sua nascita, 5.
} 
dalmatino; la quale a me è stata cara di vedere, perché egli insegna di molte cose, e della ortografia, e della proprietà della lingua, della variazion de' nomi e de' verbi.[.... E dobbiam sperar di vedere che la lingua in questo non si fermerà, che' ne avremo anche degli altri scrittori. ${ }^{62}$

Albino Zenatti definisce il libro come un semplice saggio di molte lettere inedite.

Complessivamente ne contiene 24, scritte dopo i sessant'anni, quando in seguito a una gravissima malattia, avvenne quella profonda conversione dell'animo del Muzio, trasformandolo in un dotto e serissimo moralista e teologo. Leggendo la sua biografia è chiaro che fu amico di vescovi, di patriarchi, familiare di cardinali e di papi, di due santi, per la precisione di Carlo Borromeo e Pio $\mathrm{V}^{6}$.

Tra i testi più interessanti del volume si possono considerare quelli che compose a Roma, tutto impegnato a rivedere e a rassettare i grandi scrittori al servizio della censura ecclesiastica. Il suo intento fu quello di salvare il più possibile e il meglio di scrittori e poeti. Questo incarico era assegnato il più delle volte ai frati, con i quali Muzio non aveva un buon rapporto.

Nella sua opera le Battaglie, riconobbe ogni grazia dello stile al Boccaccio, sulla quale non volle metterci sopra le mani. Lo fece invece nei Discorsi e nell'Arte della guerra di Machiavelli. Pur apprezzandolo meno come scrittore, $\mathrm{Mu}$ zio si dimostrò comunque diligente e prudente nel "sistemare" il suo lavoro visto che si rendeva conto della "sconvenienza" e della "sconcezza" di questi procedimenti. ${ }^{64}$ Scriveva al Duca d'Urbino:

Ho pensato che essi danno questa impresa ad alcuno, la potranno dare a qualche parte che ne farà quello che ha fatto un altro delle novelle del Boccaccio, il quale da un fra-

62 Muzio, Lettere inedite di Girolamo Muzio Giustinopolitano: pubblicate nel IV centenario della sua nascita, I I.

63 Zenatti, Lettere inedite di Girolamo Muzio Giustinopolitano: pubblicate nel IV centenario della sua nascita, 8-9.

64 Benedetto Croce, "Scritti di storia e politica," in Poeti e scrittori del pieno Rinascimento. Vol. I (Bari: Laterza, 1945), 205. te è stato non, come dicono, censurato ma tonsurato, in maniera che lo hanno fatto rimaner toso: ché ne ha levate ventisei novelle intere e molti squarci soppresso qua e là. E poi lo hanno dato a me, che debba rassettarlo e legarlo assieme là dove essi lo hanno guasto e disciolto! Dalla qual cosa io non sono per prenderne fatica alcuna, se non mi rimettono la impresa a me di correggerlo in tal modo, che lo netterà di tutte le cose che sono dette con poca reverenza di Dio; e per sodisfare ancora loro, leverò via tutte quelle cose che in generale sono dette contro i frati; che se si volesse levar tutto quello che è detto contro ogni particolare, ci resterebbe poco da leggere di quel libro! ${ }^{65}$

-

Nelle lettere indirizzate al duca è curiosa la profonda confidenza con la quale scrive al suo $\mathrm{Si}$ gnore. In ogni modo la lista dei principi che giovarono e si affezionarono al Muzio, a cominciare dal Marchese del Vasto, per finire con Francesco de' Medici, fu molto lunga. Ai lettori capodistriani saranno sicuramente più interessanti e care le lettere che scrisse al nipote Maurizio, nelle quali Muzio difese il suo poema, L'Egida, scritto in gloria di Capodistria. L'autore era dispiaciuto al notare che la sua opera era poco apprezzata dai suoi concittadini. L'Egida, secondo Albino Zenatti, si staccava dai soliti poemi dell'epoca ed era la dimostrazione del profondo affetto che Muzio nutriva per la sua terra. ${ }^{66}$

\section{La lirica, la poetica, il poema epico e gli scritti} sul comportamento

Al centro della lirica di Muzio si colloca il suo amore per la bella e raffinata cortigiana Tullia d'Aragona a cui dedica sonetti, canzoni e parecchie egloghe.

Le Egloghe ${ }^{67}$ sono divise in cinque libri: Le Amorose, Le Marchesane, Le Illustri, Le Lugubri,

65 Muzio, Lettere inedite di Girolamo Muzio Giustinopolitano: pubblicate nel IV centenario della sua nascita, 30-3I.

66 Zenatti, Lettere inedite di Girolamo Muzio Giustinopolitano:pubblicate nel IV centenario della sua nascita .

67 Componimento poetico solitamente di argomento pastorale o di forma amebea. 
le Varie. Nelle Amorose celebra Tullia d'Aragona con "espressioni teneramente avanzate" prima col nome di Tirennia, esaltata anche nelle sue Rime, più di quel che aveva fatto Bernardo Tasso, poi, invece, sotto il nome di Talia. ${ }^{68}$

Lo afferma pure lui stesso in una lettera a un amico:

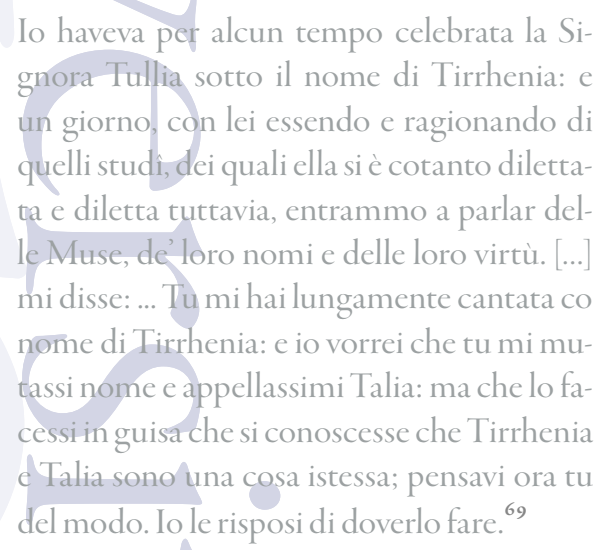

Le egloghe del secondo libro parlano del marchese Alfonso d'Avalos, dei mali della guerra, degli amori del marchese e di sua moglie. Il terzo libro tributa onori ai suoi protettori. Il quarto contiene egloghe funebri che sono le più commoventi: piange la morte di Luigi Gonzaga, deplora la scomparsa di vari suoi protettori e amici, si lamenta per la morte di Argia, sorella di Tullia d'Aragona e, infine, della sua amante Chiara, sotto il nome di Clori, celebrata già in vita.

Nelle Rime diverse, che paragona alle Odi di Orazio, celebra, con canzoni e sonetti, le sue "due donne molto amate", "l'una d'umili condizioni”, Chiara, l'altra, invece, la celebre Tullia d'Aragona.

Nell'Arte poetica, opera divisa in tre libri e molto lodata dal Varchi, l'autore volle imitare l'Arte poetica di Orazio. Nel primo libro attacca coloro che sostengono che per scrivere poesie basti "il naturale" e che non è un'arte che si possa imparare; nel secondo spiega come dare grandezza alle cose "minute", mettendo al primo posto

\footnotetext{
68 Stancovich, "Biografia degli uomini distinti dell' Istria," 7 I.

69 Muzio, Lettere inedite di Girolamo Muzio Giustinopolitano: pubblica te nel IV centenario della sua nascita, I I.
}

Virgilio davanti a Omero e Ovidio; infine, nel terzo parla della lingua italiana, dei diversi tipi di poesia, dello stile e delle metafore. ${ }^{\circ}$

L'Egida (1572), invece, è un poema eroico in dieci libri in versi sciolti, nel quale narra, usando pure elementi mitologici, la favolosa origine di Capodistria; con quest'opera celebra quella che considerava la sua città.

Muzio si occupò anche di norme sociali. La lingua italiana da lungo tempo ha una parola specifica per definire il complesso delle regole per il corretto comportamento sociale, quella di "galateo" che richiama il titolo dell'opera più famosa di Giovanni della Casa, il Galateo, overo de' costumi. In quest'opera con chiari esempi si era proposto il modello di ciò "che si convenga di fare per poter, in comunicando et in usando con le genti, esser costumato e piacevole e di bella maniera".

Le opere che Muzio scrisse su questo tema sono: Il Gentiluomo che consiste in tre dialoghi e parla della nobiltà: come si acquista, conserva e perde; inoltre da dove deriva. Distingue la nobiltà degli uomini, delle donne, delle persone "private" e "de' signori", nonché quella delle armi e delle lettere e si pone il problema quale sia la "maggiore". Scrisse, inoltre, l'operetta il Cavaliero, le Operette morali e gli Avvertimenti morali. ${ }^{73}$

\section{Conclusione}

Dopo una lunga e intensa vita, passata al servizio di numerosi uomini importanti, dopo aver stilato una miriade d'opere, seppur continuamente in viaggio, a causa di diversi incarichi che gli erano stati assegnati, si ritrovò alla fine malato, senza mezzi, abbandonato da tutti, ospite di un amico. La sua amarezza traspare chiaramente in una lettera, scritta poco prima di morire:

\footnotetext{
$70 \quad$ Stancovich, "Biografia degli uomini distinti dell’ Istria," 73-75.

71 Il poemetto narra la contesa tra Poseidone e Pallade Atene e la trasformazione dello scudo (o dell' "egida”) della dea, caduta in mare, nell' isoletta su cui sarebbe sorta Capodistria.

72 Inge Botteri, Galateo e Galatei (Roma: Bulzoni, I999), I I.

73 Stancovich, "Biografia degli uomini distinti dell'Istria," 8I.
} 
Io sto alcuna volta pensando alla mia passata vita, et alle Corti de' Prencipi dove io sono vivuto, et che ' I Marchese del Vasto mi mandò al Duca Hercole di Ferrara, et che'i Duca d'Urbino mi tolse a Don Ferrando, et che Papa Pio quarto, et poi il quinto mi tolsero al Duca d'Urbino; le quali cose mi persuadono che io sia stato qualche cosa: ma, vedendo poi che mi hanno lasciati povero, entro in altra opinione. Considerando poi che infino ad hor quindici volumi di mie opere con approbation si leggono, et che di cose cavalleresche, di morali, et di catholiche non so se altri habbia scritto più cose (per non dir meglio) di me, ritorno in fantasia di meritar pur qualche cosa. Et, se ben non fossi hora più atto alle fatiche; come soldato veterano dovrei almen essere mantenuto, come si fa alcuna volta de' cani et de' cavalli, che si pascono et si da' loro sepoltura. ${ }^{74}$

La sorte degli uomini di cultura nel Cinquecento era ingrata. Costretti a cercare i favori dei nobili facoltosi, erano sempre in balia degli umori dei loro mecenati.

Girolamo Muzio lasciò un'impronta nella storia letteraria del suo secolo e anche nella lingua italiana. Ciò gli valse onori e celebrità, ma non lo risparmiò dalle vicissitudini della vita. La sua opera più importante, Il Duello, contribuì non poco alla diffusione della lingua letteraria volgare. Assieme a Machiavelli che scrisse $I l$ principe contribuì in maniera fondamentale alla diffusione di un genere letterario di carattere politico-scientifico.

\section{Povzetek}

V široki panorami istrskih avtorjev je nemogoče obiti Girolama Muzia, Koprčana po duši in po družinskem izvoru. Gre za po krivici podcenjenega avtorja, ki je bil pozabljen zaradi svoje politične naklonjenosti Cerkvi in svojega boja za protireformacijo. »Kladivo za krivoverce $\ll$, kot je bil imenovan, se na prvi pogled predstavi kot manj privlačna figura od Vergerija in ostalih, ki pa so se približali protestantski reformi. Muzio je bil oseb-

74 Zenatti, Lettere inedite di Girolamo Muzio Giustinopolitano: pubblicate nel IV centenario della sua nascita, 74 . nost, ki bi jo lahko danes definirali kot »človek režima«, ker je bil lojalen do oblasti, ki so takrat vladale; resnici na ljubo pa bi ga lahko imeli tudi za laskača. Sredi i6. stoletja se uveljavi v vlogi dvornega privrženca in učenjaka, prav v času, ko je dvor vedno bolj postajal sedež politične moči. Tako je žal zastrt lik izobraženca kot učitelja civilizirane družbe. Muzio je izvrševal različne naloge kot tajnik v službi enega ali drugega gospodarja, čeprav je bil vedno podložen najvišji avtoriteti. Ker je obiskoval različne dvore in srečeval različne ljudi, je kmalu postal velik poznavalec viteških spretnosti, tako da so se aristokrati iz različnih krajev Evrope obračali nanj za nasvete. Njegova literarna produkcija je bila zelo obširna. Pisal je o najrazličnejših vsebinah, o veri, o vprašanju jezika - kar je bilo poglavitnega pomena v tistem času - in o pravilih bontona. Ob tem se je posvečal tudi pisanju poezije, pisem in se preizkusil tudi v epiki, ki pa jo je kasneje opustil. Delo, po katerem je zaslovel po celi Evropi, je nosilo naslov: IlDuello (Dvoboj).

Ključne besede: Girolamo Muzio, protireformacija, koprski učenjaki, »kladivo za krivoverce «, Dvoboj.

\section{Summary}

In the vast panorama of the Istrian authors, it is impossible not to discuss Girolamo Muzio, a Capodistrian by family and affection. This is an unjustly underestimated author, who pays for his political support to the curia and his struggle for reform. The "hammer of the heretics", as he was called, is a figure that may at first seem less fascinating than Vergerio and those who took charge of Protestant reform. Muzio was a man who could today be defined as a person "of the regime", loyal to the structures in power at the time; in reality he can be called a flatterer.

Towards the middle of the sixteenth century, he found himself in the typical position of a servant and literate at a time when the court was becoming more the centre of political power, thus causing a crisis for an intellectual who was seen as a master of civilisation; Muzio conducted different functions as a secretary at the service of one or other master, although always subservient to the ultimate authority. Visiting courts and meeting many people, he became particularly skilled in chivalrous matters, so noblemen from all over Europe consulted him to resolve various issues. His literary production was extensive. He dealt with diverse fields, writing on religion, 
on the question of language - which was becoming of primary importance in the period - and on rules of etiquette. In addition, he cultivated the lyric, an epistolary form, and also experimented with an epic, which he later abandoned. The work that made him famous throughout Europe was the treatise entitled: IlDuello (The Duel). Keywords: Girolamo Muzio, Counter-Reformation, Capodistrian writers, the "hammer of the heretics", The Duel.

\section{Bibliografia}

Bonora, Ettore. "Il classicismo dal Bembo al Guarini." In Storia della Letteratura Italiana. Il Cinquecento. Vol. IV. Milano: Garzanti, 1966.

Borsetto, Luciana. “Introduzione”. In Muzio Girolamo: Lettere. (Ristampa anastatica dell'edizione Sermartelli I590). Bologna: Forni, 1985.

Borsetto, Luciana. "L'Ufficio di scrivere 'in suggetto di honore'. Girolamo Muzio 'duellante' 'duellista'." Acta Histriae, IX (2000): I39-158.

Botteri, Inge. Galateo e Galatei. Roma: Bulzoni, 1999.

Cherini, Aldo e Paolo Grio. Le famiglie di Capodistria: notizie storiche ed araldiche. Trieste: Famea Capodistriana, 1998.

Croce, Benedetto. "Scritti di storia e politica." In Poeti e scrittori del pieno Rinascimento. Vol. I. Bari: Laterza, 1945.

Di Brazzano, Stefano. "Girolamo Muzio e Pietro Bonomo.” In Atti e memorie della Società Istriana di Archeologia e Storia Patria. Vol. 99. Trieste, 1999.

Dionisotti, Carlo. "La letteratura nell'età del Concilio di Trento.” In Geografia e storia della letteratura italiana. Torino, 1967.

Erspamer, Francesco. La biblioteca di Don Ferrante. Duello e onore nella cultura del Cinquecento. Roma: Bulzoni, 1982.

Giaxich, Paolo. Vita di Girolamo Muzio. Trieste: J. Papasch\&Tip., I847.

Givanardi, Claudio. La teoria cortigiana. Roma: Bulzoni, 1998.
Maier, Bruno. “Girolamo Muzio.” In Enciclopedia monografica del FriuliVenezia Giulia. Udine: Istituto per l'enciclopedia del Friuli - Venezia Giulia, 1979.

Manzoni, Alessandro. I Promessi Sposi. Milano: Principato, 1976.

Miculian, Antonio. "Contributo alla storia della riforma protestante in Istria.” In Atti del Centro di Ricerche storiche. Vol. X. 215230. Rovigno: Centro di Ricerche Storiche, 1980.

Miculian, Antonio. "La controriforma in Istria: Il concilio di Trento e il ruolo dei gesuiti." In Atti del Centro di Ricerche storiche. Vol. XXIX, 200-226. Rovigno-Trieste: Centro di Ricerche Storiche di Rovigno, 1999.

Miculian, Antonio. "Il Santo Ufficio e la riforma protestante in Istria." In Atti del Centro di ricerche storiche, Vol. XI, 215230. Rovigno-Trieste: Centro di Ricerche Storiche di Rovigno, 1980-8I.

Monti, Silvana. L'età del Rinascimento. Palermo: Palumbo, i979.

Morpurgo, Alessandro. "Girolamo Muzio. Lettura tenuta nel Gabinetto di Minerva." Estratto all'Archeografo Triestino, vol.XIII, fasc.I. Trieste: Archeografo Triestino, I893. Muzio, Girolamo. Lettere. Venezia: Gioito, I55I. Muzio, Girolamo. Battaglie di Hieronimo Mutio giustinopolitano, per diffesa dell'italica lingua, con alcune lettere a gl'infrascritti nobili spiriti. Venezia, 1582.

Muzio, Girolamo. Il duello del Mutio Iustinopolitano, con le risposte cavalleresche. Venezia: Compagnia degli Uniti, 1585.

Muzio, Girolamo. Lettere inedite di Girolamo Muzio Giustinopolitano: pubblicate nel IV centenario della sua nascita. Capodistria: Comune, i846.

Muzio Girolamo. Lettere. Rist.anastat. Bologna: Fomi, 1985.

Pagine istriane n.6. "Il Muzio grammatico, secondo un libro recente." Capodistria, 1909. 
Paschini, Pio. "Episodi della Controriforma in lettere inedite di Girolamo Muzio." In Atti e Memorie della società istiana di archeologia e storia patria, XXXIXfascicolo I, 354-36I, Trieste: Società istriana di archeologia e storia patria, 1927.

Pigna, Giovan Battista. Il duello. Venezia: Valgrisi, 1554 .

Salimbeni, Fulvio. "Fonti e studi sulla storia religiosa dell'Istria nel XVI secolo." In L'umanesimo in Istria, cur. Vittore Branca e Sante Graciotti. 165-179. Firenze: Leo S. Olschki, 1983.

Semi, Francesco. Istria e Dalmazia. Uomini e tempi. Istria e Fiume. Vol. I. Udine: Del Bianco, I99I.

Stancovich, Pietro. "Biografia degli uomini distinti dell'Istria." In Atti del Centro di Ricerche Storiche di Rovigno, Vol.3. Rovigno-Trieste: Centro di Ricerche Storiche di Rovigno, 1972.

Zenatti, Albino. Lettere inedite di Girolamo Muzio Giustinopolitano: pubblicate nel IV centenario della sua nascita. Capodistria: Comune, I846. 